\title{
A VIOLÊNCIA DA CONTRARREFORMA PSIQUIÁTRICA NO BRASIL: UM ATAQUE À DEMOCRACIA EM TEMPOS DE LUTA PELOS DIREITOS HUMANOS E JUSTIÇA SOCIAL
}

\section{The violance of the counter-psychiatric reform in Brazil: on attack on democracy in times of struggle for Humanos Rights and social justice}

Ana Maria Fernandes Pitta (UCSal)

Ana Paula Guljor (Escola Nacional de Saúde Pública)

Informações do artigo

Recebido em 30/04/2019

Aceito em 03/05/2019

doi>: https://doi.org/10.25247/2447-861X.2019.n246.p6-14

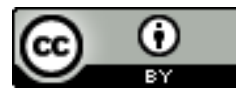

Esta obra está licenciada com uma Licença Creative Commons Atribuição 4.0 Internacional.

Como ser citado (modelo ABNT)

PITTA, Ana Maria Fernandes; GULJOR, Ana Paula. A violência da contrarreforma psiquiátrica no Brasil: um ataque à democracia em tempos de luta pelos direitos humanos e justiça social. Cadernos do CEAS: Revista Crítica de Humanidades, Salvador, n. 246, jan./abr., p. 6-14, 2019. DOI: https://doi.org/10.25247/2447$861 X .2019 \cdot 1246 \cdot \mathrm{p6}-14$

\begin{abstract}
Resumo
A Reforma Psiquiátrica brasileira é um processo iniciado em fins da década de 70, cujo desencadear foi originado nas condições de violação de direitos em grandes instituições psiquiátricas do país e na ineficácia do isolamento como estratégia terapêutica. Nos últimos 40 anos, consolidou-se como uma política de Estado e, neste sentido, entre suas diretrizes fundamentais está o cuidado em liberdade e a promoção do protagonismo social, autonomia e reinserção social das pessoas em sofrimento mental. Foi criado um arcabouço complexo de políticas voltadas para a construção de novos dispositivos de cuidado associadas a estratégias intersetoriais voltadas à superação de um modelo que historicamente se centrava em estruturas hospitalares e tinha como pressuposto a doença como principal foco de intervenção, o qual gerou cronificação, institucionalização e exclusão dos internados de seus vínculos societários. $O$ artigo se propõe a contribuir para o debate urgente e necessário ao enfrentamento de retrocessos e fortes ameaças a tudo que se conquistou no campo dos Direitos Humanos dos usuários de instituições psiquiátricas.
\end{abstract}

Palavras-chave: Direitos Humanos. Reforma Psiquiátrica. Políticas de Saúde Mental.

\section{Abstract}

The Brazilian Psychiatric Reform is a process initiated in the late 1970s, which originated in the conditions of violation of rights in large psychiatric institutions and the ineffectiveness of isolation as a therapeutic strategy. In the last 40 years it has consolidated itself as a State policy and, in this sense, among its fundamental guidelines is care in freedom and the promotion of social protagonism, autonomy and social reintegration of people in mental suffering. A framework has been created a complex of policies aimed at the construction of new care devices associated with intersectoral strategies aimed at overcoming a model that historically focused on hospital structures and assumed the disease as the main focus of intervention, which generated chronification, institutionalization and exclusion of internees of their corporate ties. The article proposes to contribute to the urgent and necessary debate to face setbacks and strong threats to everything that has been achieved in the field of Human Rights of users of psychiatric institutions.

Keywords: Human Rights. Psychiatric Reform. Mental health policies. 
Para um movimento que se tornou um modelo nacional e internacionalmente reconhecido como uma importante contribuição ao processo de desinstitucionalização de enfermos mentais no mundo, A Reforma Psiquiátrica brasileira, que acontece no momento da democratização do país, desde os 21 anos de ditadura militar a que o Brasil esteve submetido, assiste agora ao desmonte da política de Saúde Mental e uso prejudicial de Drogas.

Superar o preconceito e a marginalidade que tem caracterizado o modo de lidar com a loucura na sociedade brasileira requer que tenhamos uma sociedade justa, democrática e com oportunidades para todos. Num decálogo de princípios básicos para a existência de uma rede substitutiva aos manicômios, o movimento da Reforma Psiquiátrica brasileira tem prescrito:

1. Que a rede de cuidados seja comunitária e de fácil acesso, multiprofissional e resolutiva para a maioria dos problemas de saúde mental e uso prejudicial de álcool e outras drogas;

2. Que seja municipal e articulada à atenção básica de saúde com equipe mínima de 1psicólogo, 1 assistente social, 1 médico treinado em Saúde Mental, para populações de até 15.000 habitantes.

3. Que tenha uma retaguarda de Centros de Atenção Psicossocial, potentes e resolutivos com equipes multiprofissionais de mais de 5 profissionais qualificados (Psicólogos, Psiquiatras, enfermeiros, assistentes sociais, terapeutas ocupacionais, educadores físicos, oficineiros, agentes comunitários e outros) com leitos de suporte à crises para populações acima de 15.000 habitantes.

4. Que o cuidado mental esteja verdadeiramente incluído no sistema de atenção da Rede de Urgências e Emergências (SAMU, Unidades de Pronto AtendimentoUPA, Pronto Socorro-PS), com equipes de referência contratadas;

5. Que os Hospitais Gerais e maternidades estejam preparados para atender à integralidade somática e psíquica da clientela com interconsultas clínicopsicológica e internação psiquiátrica de emergência. Especial cuidado para crianças, adolescentes e idosos que deverão estar em hospitais gerais e/ou pediátricos se necessário. 
6. Que haja supervisão clínico-institucional constante, instituindo um permanente programa de enfrentamento da cronificação de equipes e serviços, apoiando e empoderando as equipes e por consequência usuários e familiares por eles cuidados para os enfrentamentos cotidianos da vida.

7. Que os tratamentos sejam o menos invasivo possível, estando proscrita a eletroconvulsoterapia, uma terapêutica restrita.

8. Que sejam implementados programas de uso racional de psicofármacos associados a gestão autônoma de medicação pelos usuários.

9. Que a Reabilitação Psicossocial com seu potencial de recuperação e superação de desvantagens advindas de transtornos mentais ou uso abusivo de substâncias psicoativas esteja presente em todas as fases dos tratamentos com serviços residenciais, educação inclusiva, habilitação laboral, de arte e lazer.

10. Que Programas de Cooperativas Sociais e Economia Solidária possam estar articulados às Redes de Atenção Psicossocial para garantia de trabalho e sobrevida digna aos usuários, em liberdade.

Pautado nestes princípios, o respeito a pessoa em sofrimento e o cuidado pautado na liberdade como orientadores das ações, a internação psiquiátrica não mais se constitui como recurso patognomônico de tratamento. Ou seja, nenhum fenômeno psíquico isoladamente constitui indicativo de internação.

No campo dos dispositivos assistenciais, os Centros de Atenção Psicossociais têm desempenhado uma função estratégica na continência de situações de crise, com leitos de acolhimento (CAPS III) voltados para usuários em graves sofrimento psíquico e usuários abusivos de drogas.

As Residências Terapêuticas destinadas aos egressos de internações psiquiátricas de longa permanência ou mesmo para aqueles que, sem internações prévias, mas cujos vínculos sociais foram desfeitos ao longo dos anos, necessitam de uma moradia protegida e supervisionada, onde possam receber cuidados de um lar, com famílias substitutas que os permitam viver em liberdade. Os leitos psiquiátricos em hospitais gerais entre outros, são estruturas fundamentais no modo da atenção psicossocial, como modelo substitutivo ao 
hospital psiquiátrico nas situações agudas de emergência, garantindo os cuidados clínicos intensivos que hospitais psiquiátricos não costumam oferecer.

A promulgação da lei 10.216 em 06 de abril de 2001 se configurou em um marco orientador dos direitos dos usuários no campo da saúde mental e fruto de uma ampla discussão da sociedade e mobilização de usuários, familiares e profissionais.

No curso dos últimos 18 anos de aprovação da lei, a rede de suporte intersetorial em saúde mental desenvolveu uma ampla gama de estratégias substitutivas a internação psiquiátrica, pois a transformação epistemológica da concepção de 'crise' efetuou uma ruptura conceitual com a noção de que esta situação se restringe a alterações psicopatológicas restrita ao processo natural de 'doença'. A situação de crise deve ser contextualizada com a rede de apoio social do usuário, sua vulnerabilidade e vínculos sociais.

A internação psiquiátrica é um recurso de exclusão, isto é, diferentes abordagens comunitárias devem ser realizadas antes de se decidir internar alguém. E assim está descrito na lei nacional, em seu artigo $4^{\circ}$ : "A internação, em qualquer de suas modalidades, só será indicada quando os recursos extra hospitalares se mostrarem insuficientes."

A internação psiquiátrica deve ser considerada um recurso terapêutico com forte potencial iatrogênico, na medida em que é um procedimento que induz à recorrência (reinternações), pior prognóstico a longo prazo de transtornos psiquiátricos, aumento desproporcional para o custo do sistema de saúde e da assistência além de promoção de estigma, isolamento e fragilização das relações sociais dos hospitalizados.

A complexidade da rede de dispositivos existentes hoje, apesar da distribuição não ser equilibrada em todo o território nacional e necessitar de maiores investimentos para sua ampliação, tem demonstrado sua efetividade através da diversidade de ofertas de recursos para lidar com as questões envolvidas nas 'crises' recebidas em seus dispositivos. A manutenção dos vínculos comunitários gera a preservação dos laços de afetividade e, quando o cuidado intensivo se fizer necessário ocorrer, contar com suporte das equipes no período noturno é efetivado no Centro de Atenção Psicossocial com leitos de acolhimento no próprio território do usuário, preservando a relação com sua equipe clínica de referência.

A existência de quadros onde são necessários suportes de clínica médica, como na questão envolvendo álcool e outras drogas, podem ser acolhidos em leitos de hospitais gerais, cuja proporção de leitos de enfermaria é reduzida em comparação aos hospitais psiquiátricos e possibilitam o cuidado individualizado através de projetos terapêuticos 
singularizados. Estas internações acontecem por períodos curtos, não produzindo institucionalização e preservando o vínculo de acompanhamento com a equipe de referência do CAPS, seja para os usuários do CAPS para álcool e drogas ou de transtornos mentais.

A Reforma Psiquiátrica Brasileira nasce do trabalho singular de pessoas como Nise da Silveira, Luís da Rocha Cerqueira e Osório Cesar, desde o início do século passado, ganhou força no final dos anos 1970, com o Movimento dos Trabalhadores de Saúde Mental, que cresceu com o Movimento da Luta Antimanicomial na segunda metade dos anos 1980, e a Reforma Psiquiátrica foi gradualmente se transformando em política oficial do Estado brasileiro.

A partir de 1990, atravessando governos ideologicamente distintos, mas todos percorrendo os caminhos da redemocratização do país, fomos assistindo às lutas de profissionais, usuários, familiares, juristas e alguns parlamentares contra as estratégias de hospitalização e enclausuramento dos "loucos de todo o gênero" em manicômios psiquiátricos, política pública então vigente. A Coordenação de Saúde Mental do Ministério da Saúde foi sucessivamente ocupada por militantes da Reforma Psiquiátrica, fazendo avançar, na dependência das conjunturas, favoráveis ou não, uma reforma da assistência desospitalizante, dirigida ao território, à comunidade, num exercício ativo do cuidar em liberdade, seguindo o princípio de que a liberdade é terapêutica!

Esse movimento, que sempre foi de difíceis conquistas, mas de consistentes avanços, começa a sofrer ataques no final de 2015 , em sintonia com as mudanças e os retrocessos na vida política brasileira. A nomeação de um diretor do maior asilo manicomial da América Latina, nos anos de chumbo da ditadura militar brasileira, quando a política prescrita para o setor era o enclausuramento e a cronificação dos usuários, desencadeou protestos em muitos lugares do país, com a "ocupação" da Coordenação de Saúde Mental em Brasília durante mais de três meses. Saiu em maio de 2016, junto ao ministro amigo, que o tinha alçado ao cargo sem atributos ou competências que o legitimassem.

Após uma vacância de 9 meses na Coordenação Nacional da Saúde Mental, a maré da direitização das políticas da contrarreforma é reforçada pela indicação de representante da Associação Brasileira de Psiquiatria, que, no passado, foi uma grande aliada da Reforma na defesa de Leis e políticas que a respaldassem, passou a ser, nas últimas décadas, defensora dos maiores retrocessos deste vitorioso movimento de Direitos humanos e inclusão social 
dos usuários de instituições psiquiátricas segregadoras, hegemônicas nos momentos de frágil democracia no país.

Uma política e leis construídas no mais intenso debate onde todos os setores estiveram participando até a sua aprovação vêm, desde dezembro de 2017, ser aprovada, sem a participação dos interessados, a denominada "nova política de saúde mental" que, de novo, traz apenas o retorno à hospitalização para usuários com transtornos mentais em hospitais psiquiátricos, e, em Comunidades Terapêuticas, para os usuários de álcool e outras drogas, multiplicando as camas de internação a números superiores aos tempos da "Indústria da Loucura", quando o país gastava 97\% dos recursos para o setor em hospitais (1982) e apenas 3\% em estruturas comunitárias.

Também a rede ambulatorial retorna ao modelo de prescrição de psicofármacos e guias de internação, quando já havia evoluído para estruturas comunitárias mais potentes, que podem garantir até hospitalizações, se o Plano Terapêutico assim o requeira. A Nota Técnica sequencial publicada em dezembro de 2018 inclui o hospital psiquiátrico na Rede de Atenção Psicossocial, com ajuste do valor das AlHs.

O dinheiro do orçamento público, que havia progressivamente migrado para a rede de atenção psicossocial comunitária, volta para a rede hospitalar e de comunidades terapêuticas igualmente enclausuradoras, sem a resolutividade dos problemas humanos ali confinados. Afinal, a vida é crônica. Segue, após qualquer tipo de interrupção nos processos de internação, e as pessoas precisam serem assistidas em serviços territoriais resolutivos para seguirem vivendo suas vidas! Também os leitos psiquiátricos e de Álcool e Drogas em Hospitais Gerais, como previsto na Lei 10.216, vão perdendo a importância nessas novas decisões.

Assistimos, assim, a uma brutal reversão da política exitosa prescrita em Lei, que necessita o incremento de novos recursos para torná-la mais potente e resolutiva, ser autoritariamente golpeada. O documento "Esclarecimentos sobre as mudanças na Política Nacional de Saúde Mental e nas Diretrizes na Política Nacional sobre Drogas", retirado do ar após uma enxurrada de críticas recebidas, sacraliza "mudanças" que apenas dão substantivos passos atrás naquilo que o país vem se esforçando para superar.

Desta forma, o documento parece ignorar que foi a ênfase em serviços substitutivos aos hospitais psiquiátricos, garantindo o acesso a tratamento em liberdade e próximo ao local de moradia de usuários, que culminou com uma redução responsável de moradores de 
hospitais e sobrevida digna a pessoas iatrogenicamente cronificadas nas nossas instituições. Essa mudança implicou a reversão dos gastos públicos na área, jogando-se fora estratégias exitosas como tratamento assertivo na comunidade, de redução de danos, que implicam o cuidado contínuo e em liberdade, mesmo que o uso de drogas não tenha sido interrompido, com ênfase na prevenção e atenuação de agravos à saúde, utilizadas por dezenas de países em todo o mundo (BEG; STRATHDEE; KAZATCHKINE, 2015) e somente possível com uma rede comunitária de serviços solidamente estruturada para acompanhá-los.

Outro fator preocupante é a dissociação da gestão das políticas de saúde mental do Ministério da Saúde e a de álcool e outras drogas, agora na Secretaria Nacional de Cuidados e Prevenção às Drogas (SENAPRED), no Ministério da Cidadania, ministério esse fortemente comprometido com as comunidades terapêuticas com amplo envolvimento político e religioso.

Outros aspectos problemáticos, na Nota, são a defesa de ambulatórios "especializados" desconsiderando a cobertura assistencial da Atenção Primária e o matriciamento por Núcleos de Apoio à Saúde da Família - NASF, ou mesmo a rede dos mais de 2.000 CAPS espalhados no território nacional, garantindo, assim, atenção integral e descentralizada no território nacional. O único momento no qual a saúde mental da infância e juventude está contemplada tem a ver com internação em hospitais.

No que diz respeito às tecnologias de cuidado, apenas a defesa da eletroconvulsoterapia (ECT) com garantia de financiamento está presente, esquecendo seu histórico de uso massivo nos manicômios brasileiros há décadas passadas, quando, mesmo a indicação como terapêutica se dá aos raros casos em que as demais estratégias farmacológicas e psicoterápicas não alcançam bom êxito nas depressões graves ou severas. Como retorno ao primitivo, o ECT ainda está envolto em controvérsias a respeito de sua eficácia e efeitos colaterais (REISNER, 2003). Sem justificativa, a utilização e o financiamento generalizado de um procedimento para uso tão raro, quando o acesso aos demais, mais frequentes, não estão garantidos.

A publicação deste número dos Cadernos do CEAS, privilegiando artigos no campo dos Direitos Humanos, Saúde Mental e Drogas, no mês da Luta Antimanicomial e em tempos de grande reação crítica dos movimentos sociais aos desmandos e retrocessos impostos pelos atuais dirigentes das políticas específicas e gerais no país, é para celebrar. Artigos oriundos de Fóruns Brasileiros de Direitos Humanos e Saúde Mental da Associação Brasileira 
de Saúde Mental, juntados a outros artigos de pesquisadores interdisciplinares ventilam o debate oportuno que o momento atual, de perdas e retrocessos, somente nos instiga!

\section{Referências}

\section{ASSOCIAÇÃO BRASILEIRA DE SAÚDE COLETIVA. Sobre as mudanças na Política} Nacional de Saúde Mental e nas Diretrizes da Política Nacional sobre Drogas. Disponível em:< https://www.abrasco.org.br/site/outras-noticias/saude-da-populacao/sobre-asmudancas-napolitica-nacional-de-saude-mental-e-nas-diretrizes-da-politica-nacionalsobre-

drogas/39619/?fbclid=IwARorBI8RhgarMfFGu2G6V_a2OK3fP449bE3 YoxtZHAD6gT5AdrnF OU 4 ac>. Acesso em: 24 fev. 2019.

BEG, M.; STRATHDEE, S. A.; KAZATCHKINE, M. State of the art science addressing injecting drug use, HIV and harm reduction. International Journal of Drug Policy, v. 26, suppl. 1, p. S1-S4, 2015.

BRASIL. Ministério da Saúde. Saúde Mental em Dados 12, v. 10, n. 12, out. 2015. Informativo eletrônico. Disponível em: Acesso em: 19 fev. 2019.

CAMARGO JR., K. R. Saúde mental, cidadania e o campo científico. Physis: Revista de Saúde Coletiva. Rio de Janeiro, v. 25, n. 4, p. 1057-1058, 2015.

CONSELHO ESTADUAL DE SAÚDE DE PERNAMBUCO. CFP- Relatório da Inspeção Nacional em Comunidades Terapêuticas - 2017. DF: CFP, 2018. Disponível em: Acesso em: 24 fev. 2019.

INSTITUTO DE PESOUISA ECONÔMICA APLICADA. Nota Técnica n. 21. Perfil das Comunidades Terapêuticas Brasileiras. Brasília: Diretoria de Estudos e Políticas do Estado, das Instituições e da Democracia/IPEA, março de 2017.

LIMA. RC. Contrarreforma psiquiátrica Physis: Revista de Saúde Coletiva, Rio de Janeiro, v. 29(1), e290101, 2019

REISNER, A. D. The electroconvulsive therapy controversy: evidence and ethics. Neuropsychology review, v. 13, n. 4, p. 199-219, 2003.

THORNICROFT G.; TANSELLA, M. Balancing community-based and hospital-based mental health care. World psychiatry, v. 1, n. 2, p. 84-90, 2002. 


\section{Dados das autoras}

\section{Ana Maria Fernandes Pitta}

Doutora em Medicina Preventiva/Saúde Mental (USP); professora do Programa de Pós-graduação em Políticas Sociais e Cidadania, Universidade Católica de Salvador; Presidente da Associação Brasileira de Saúde Mental. E-mail: ana.pitta@pro.ucsal.br

\section{Ana Paula Guljor}

Psiquiatra. Phd em Saúde Pública. Pesquisadora do Laboratório de Estudos e Pesquisas em Saúde Mental e Atenção Psicossocial - LAPS da Escola Nacional de Saúde Pública. Secretária da Associação Brasileira de Saúde Mental. Email: anapaulaguljor@gmail.com 